\title{
A paediatric systemic lupus erythematosus patient presenting with acute pancreatitis: a rare case
}

\author{
Ahmet Basturk ${ }^{1}$, Reha Artan ${ }^{1}$, Aygen Yilmaz ${ }^{1}$, Utku Senol ${ }^{2}$ \\ ${ }^{1}$ Department of Paediatric Gastroenterology, Faculty of Medicine, Akdeniz University, Antalya, Turkey \\ ${ }^{2}$ Department of Radiology, Faculty of Medicine, Akdeniz University, Antalya, Turkey
}

Gastroenterology Rev 2016; 11 (4): 296-298

DOI: $10.5114 / p g .2016 .61469$

Address for correspondence: Ahmet Basturk PhD, Department of Paediatric Gastroenterology, Faculty of Medicine, Akdeniz University, Akkuyu mh. 1039. sk. Onursal kop. G Blok No: 16, 07059 Antalya, Turkey, fax: +90 5304167543, e-mail: drahmetbasturk@hotmail.com

Systemic lupus erythematosus (SLE) is a disease associated with widely varying clinical presentations characterised by multisystem involvement. While gastrointestinal involvement is known as a very common situation, acute pancreatitis is a rare condition [1]. Systemic lupus erythematosus cases with acute pancreatitis have rarely been described in the medical literature [2].

The aim of the current study is to present a paediatric patient with SLE, who presented at our clinic after developing acute pancreatitis.

The patient was a 3-year-old girl who was admitted to the clinical centre with the symptoms of fever, stomach ache, fatigue, facial and eye flushing, and swelling in both ankles. The swelling in both ankles was persistent over the previous month. In the 15 days prior to presentation at our clinic, the patient had fever, stomach ache, asthaenia, and facial and eye rubor. Although her family's medical history indicated that one of her siblings had a neuromotor development disorder, the patient's medical history was unremarkable. Upon physical examination, the patient weighed $12 \mathrm{~kg}\left(10-25^{\text {th }}\right.$ percentile) and her height was $97 \mathrm{~cm}$ ( $50^{\text {th }}$ percentile). She had conjunctivitis and malar rush in both eyes, sensitivity at the epigastric area in the right upper quadrant, and arthritis in both ankles. Laboratory data of the patient were as follows: haemoglobin $(\mathrm{HB}) 7.8 \mathrm{~g} / \mathrm{dl}$ (N: 9-11), leucocyte (WBC) $2110 / \mathrm{mm}^{3}$ (N: 4.8-10.8), lymphocyte $600 / \mathrm{mm}^{3}$ (N: 1.2-3.5), thrombocyte (PLT) $195000 / \mu \mathrm{l}(\mathrm{N}: 150$ 000-450 000), alanine aminotransferase (ALT) $235 \mathrm{U} / \mathrm{l}(\mathrm{N}: 0-41)$, aspartate aminotransferase (AST) $312 \mathrm{U} / \mathrm{l}$ (N: 0-40), $\gamma$-glutamyl transferase (GGT) $243 \mathrm{U} / \mathrm{l}$ (N: 10-61), total bilirubin (T. Bil) $4.7 \mathrm{mg} / \mathrm{dl}$ (N: 0.1-1.2), direct bilirubin (D. Bil) $3.9 \mathrm{mg} / \mathrm{dl}$ (N: 0-0.2), prothrombin time (PT) $11.8 \mathrm{sn}$ (N: 11-14), INR 1.05 (N: 0.9-1.2), active partial thrombin time (aPTT) $28.2 \mathrm{sn}(\mathrm{N}$ : 25-33), lipase $1291 \mathrm{U} / \mathrm{l}(\mathrm{N}: 13-60)$, pancreatic amylase
$541 \mathrm{U} / \mathrm{l}(\mathrm{N}: 13-53)$, sedimentation $76 \mathrm{~mm} / \mathrm{h}(\mathrm{N}: 0-20)$, total protein $5.9 \mathrm{~g} / \mathrm{dl}$, and albumin $3.86 \mathrm{~g} / \mathrm{dl}$ ( $\mathrm{N}: 3.5-5)$. Serum electrolytes, kidney function tests, coagulation, lipid profile test, and peripheral smear tests were all normal. Protein (+), ketone $(++)$, and bilirubin (+) were found in urinalysis. The stool tests were negative for pathologic findings. Minimal liquor in the peripancreatic area and oedema in pancreas were observed during abdominal ultrasonography. The patient was diagnosed with acute pancreatitis and oral feeding was ceased. Lansoprazole and total parenteral nutrition were given intravenously. The aetiology of the acute pancreatitis was investigated. Magnetic resonance cholangiopancreatography (MRCP) normal image showed the common bile duct and the pancreatic duct (Figure 1). Tests done for hepatitis diseases such as hepatitis markers, toxoplasmosis, rubella, cytomegalovirus, herpes simplex, Epstein Barr virus, and parvovirus were all negative. Moreover, the total creatine kinase level was $24 \mathrm{U} / \mathrm{I}(\mathrm{N}: 0-20)$ while the $\alpha 1$ antitrypsin, ferritin, and ceruloplasmin levels were $148 \mathrm{mg} / \mathrm{dl}$ ( $\mathrm{N}:$ 20-280), $165 \mathrm{mg} / \mathrm{dl}$ (N: 20-100), and $32 \mathrm{mg} / \mathrm{dl}$ (N: 20-70), respectively. The results of immunofluorescence tests were as follows: Antinuclear antibody (ANA) 1/160 homogenous, anti-deoxyribonuclease DNA (anti-dsDNA), anti-smith, anti SS-A, and anti-SS-B were positive. Anti-ribonucleoprotein (anti RNP), anti-scleroderma 70 (anti scl 70), p-anti-neutrophil antibody ( $p$-ANCA), c-anti-neutrophil antibody (c-ANCA), anti-smooth muscle antibody (ASMA), liver/kidney microsomal antibody (anti-LKM), anti-mitochondrial antibody (AMA), lupus anticoagulant, and anti-cardiolipin IgG-IgM were negative. The C3c (34 mg/dl (N: 50-110)) and C4 (4.2 mg/dl (N: 20-50)) were low. Clinical findings included: Malar rash, arthritis, positive ANA and anti-ds-DNA reactions, haematological disorders (anaemia, leucopaenia, lymphopaenia) and posi- 
tive anti-Sm antibody. The patient was diagnosed with SLE according to the diagnostic criteria of the American College of Rheumatology (ACR). The patient also had hypocomplementaemia, anti-SS-A, anti-SS-B, and ocular inflammation. A biopsy indicated that there was no kidney involvement. Azathioprine ( $2 \mathrm{~m} / \mathrm{kg} /$ day p.o.) and methylprednisolone ( $2 \mathrm{mg} / \mathrm{kg} /$ day i.v.) were given to treat the lupus diagnosis. After 2 months of treatment, the patient recovered with complete resolution of the symptoms. Upon physical examination of the patient, the conjunctivitis, arthritis, and sensitivity at the epigastric area and right upper quadrant were absent. The results of abdominal ultrasonography were also observed as normal values. Laboratory test results were as follows: HB $11.5 \mathrm{~g} / \mathrm{dl}$, WBC 8600/mm³ $\mathrm{mm}^{3}$, lymphocyte 2300/

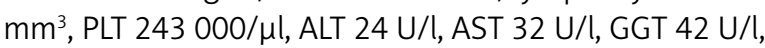
T. Bil $1.1 \mathrm{mg} / \mathrm{dl}$, D. Bil $0.2 \mathrm{mg} / \mathrm{dl}$, lipase $40 \mathrm{U} / \mathrm{l}$, pancreatic amylase $56 \mathrm{U} / \mathrm{l}$, sedimentation $16 \mathrm{~mm} / \mathrm{h}$, total protein $6.2 \mathrm{~g} / \mathrm{dl}$, albumin $4.3 \mathrm{~g} / \mathrm{dl}$, ANA and anti-ds-DNA negative, C3c $68 \mathrm{mg} / \mathrm{dl}$, and C4 $42 \mathrm{mg} / \mathrm{dl}$. All biochemical test results were within normal limits. The general condition of the patient was good and she was discharged from the hospital and followed-up in an outpatient basis. The general condition of the patient has been good for the last 12 months. In addition to these, blood tests and urinalysis results remain within normal limits. The patient is currently undergoing follow-up care for SLE at the paediatric outpatient clinic. Written and verbal consent was provided by the parents of the patient regarding the publication of the case materials.

Pancreatic involvement is rare in SLE. The most common causes of acute pancreatitis are mechanical narrowing due to obstruction of the pancreatic canal and toxic-metabolic factors such as alcohol, drugs, hypercalcaemia, or hypertriglyceridaemia. None of these conditions were present in this case [3]. The aetiology of pancreatitis in SLE is not well understood. However, it is known that auto-immune factors including vasculitis, non-inflammatory vasculopathy, or thrombosis related to anti-phospholipid antibodies may have contributed to etiopathogenesis in this case. In autopsy series, inflammatory involvement has been demonstrated to occur in many patients. Inflammation is a consequence of generalised or terminal disease. Pancreatitis in an SLE patient may be related to abnormal cellular responses caused by the autoimmune reaction or an autoimmune response resulting from generalised inflammation. Sub-clinical pancreatitis may develop in a large portion of SLE patients [4]. Saab et al. [5] examined 891 patients with SLE for 9 years, reporting 8 cases of SLE (0.1\%). Pascual-Ramos et al. [6] followed 895 patients with SLE for 17 years, observing pancreatitis in 35 (0.23\%) patients. Nearly half of these cases were attributable to

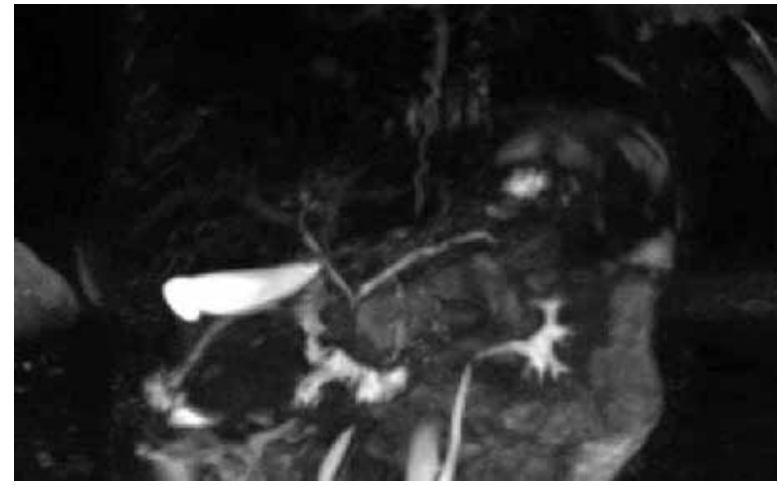

Figure 1. 3D Heavily T2-weighted normal image shows the common bile duct and the pancreatic duct

toxic and metabolic causes, and only $0.11 \%$ were diagnosed as idiopathic pancreatitis. Severe pancreatitis occurs in $15-20 \%$ of SLE patients with acute pancreatitis, and the probability of complications is very high among patients with severe pancreatitis. The mortality rate in severe pancreatitis is $30 \%$ [7]. Pancreatitis mortality rates are further elevated among patients with SLE [6].

Among patients with lupus erythematosus, high serum creatine, hypoalbuminaemia, anti-ds-DNA antibodies, thrombocytopaenia, and low complement levels are indicative of poor prognosis. In the present case, we observed positive anti-ds-DNA antibody tests and hypocomplementaemia [3].

Steroids, diuretics, and immunosuppressive agents contribute to the aetiology of SLE but they are also used in medical treatment [8]. Thus, the role of corticosteroids is difficult to ascertain due to the natural coexistence of disease activity and treatment with high doses of prednisone [6]. Initial studies support a detrimental effect of corticosteroid treatment in the treatment of pancreatic disease, including acute pancreatitis [9]. However, Saab et al. [5] published a series of 8 SLE patients with inactive disease and diagnosed with acute pancreatitis. Their biochemically and clinically acute pancreatitis episodes improved after treatment with high doses of corticosteroid. Corticosteroids with azathioprine treatment was also used in this study, and the patient responded well to the treatment.

As a result, the possibility of acute pancreatitis should be considered during the diagnostic evaluation of SLE patients with symptoms of stomachache and vomiting. In the present case, we observed acute idiopathic pancreatitis similar to other SLE patients with pancreatitis. The patient responded well to the corticosteroid azathioprine and other supplementary treatments.

\section{Conflict of interest}

The authors declare no conflict of interest. 


\section{References}

1. Wang F, Wang NS, Zhao BH, et al. Acute pancreatitis as an initial symptom of systemic lupus erythematosus: a case report and review of the literature. World J Gastroenterol 2005; 11 : 4766-8.

2. Penalva JC, Martinez J, Pascual E, et al. Chronic pancreatitis associated with systemic lupus erythematosus in a young girl. Pancreas 2003; 27: 275-7.

3. Mitchell RM, Byrne MF, Baillie J. Pancreatitis. Lancet 2003; 361: 1447-55.

4. Eberhard A, Couper R, Durie P, et al. Exocrine pancreatic function in children with systemic lupus erythematosus. J Rheumatol 1992; 19: 964-67.

5. Saab S, Corr MP, Weisman MH. Corticosteroids and systemic lupus erythematosus pancreatitis: a case series. J Rheumatol 1998; 25: 801-6.

6. Pascual-Ramos V, Duarte-Rojo A, Villa AR, et al. Systemic lupus erythematosus as a cause and prognostic factor of acute pancreatitis. J Rheumatol 2004; 31: 707-12.

7. Baron TH, Morgan DE. Acute necrotizing pancreatitis. N Engl J Med 1999; 340: 1412-7.

8. Eaker EY, Toskes PP. Systemic lupus erythematosus presenting initially with acute pancreatitis and a review of the literature. Am J Med Sci 1989; 297: 38-41.

9. Somogyii L, Martin SP, Venkatesan T, et al. Recurrent acute pancreatitis: an algorithmic approach to identification and elimination of inciting factors. Gastroenterology 2001; 120: 708-17.

Received: 29.07.2015

Accepted: 24.12.2015 